\title{
Interleukin 2 for patients with renal cancer
}

\author{
Steven A Rosenberg
}

Interleukin 2 (IL-2) is the only systemic treatment currently available that is capable of curing patients with metastatic renal cell cancer (RCC). The first indications that IL-2 could mediate objective responses in patients with metastatic RCC were reported in 1985. On the basis of the durability of the complete responses in multiple trials, the FDA approved high-dose IL-2 for the treatment of patients with metastatic RCC in 1992. In the Surgery Branch of the National Cancer Institute, a $20 \%$ objective response rate ( $9 \%$ complete and $11 \%$ partial) was seen in 259 consecutive patients with metastatic RCC treated with high-dose IL-2 alone, with only 2 treatment-related deaths. Among the 23 patients showing a complete response, disease recurrence has occurred in only 4 patients; the remaining 19 patients all have ongoing responses of between 2 and 18 years. These results indicate that over $80 \%$ of all patients who obtain a complete response following high-dose IL-2 never experience disease recurrence and seem to be cured of their disease.

In the August 2007 issue of this journal, two Practice Point articles reviewed the recently published studies that evaluate the multikinase inhibitors sorafenib and sunitinib for the treatment of patients with metastatic RCC. Treatment with these agents resulted in the prolongation of disease-free survival, and on this basis sorafenib and sunitinib were approved by the FDA. There were no complete responses in the 335 patients who received sunitinib and one complete response in the 451 patients who received sorafenib.

In the commentary on the sorafenib paper, Twardowski and Figlin appropriately concluded that sorafenib is "primarily indicated as a second-line treatment for patients...who develop progressive disease after cytokine therapy". In the commentary of the paper on sunitinib, however, Stadler and Szmulewitz concluded that sunitinib is a "reasonable
Decisions

about the use

of IL-2 seem

to be more

related to the

training of

oncologists

than to the

effectiveness

of the

treatment.

SA Rosenberg is an Advisory Board member of Nature Clinical Practice Oncology.

\section{Competing interests}

The author declared no competing interests.

www.nature.com/clinicalpractice doi:10.1038/ncponc0926 first-line agent, especially in patients with good or intermediate prognosis, and is a standard of care". They base this statement on the comment that IL-2 is "toxic and not feasible for most patients". Stadler and Szmulewitz, therefore, advocate the use of sunitinib, despite the fact that this agent cures virtually no one, whereas high-dose IL-2 apparently cures about $8 \%$ of patients.

High-dose IL-2 has been considerably underused in the treatment of patients with metastatic RCC, because it is inconvenient to administer and results in types of toxicity not common in the practice of medical oncologists. It must be administered as an inpatient procedure at 8-hour intervals and each dose needs to be administered to patient tolerance. The major toxicities of high-dose IL-2 result from a capillary-leak syndrome, which leads to fluid extravasation into tissue, hypotension and oliguria, all of which are reversible with no longterm sequelae. Physicians with experience in the use of high-dose IL-2 can very safely administer this agent to patients with normal cardiac and renal function.

Careful monitoring and fluid replacement can solve virtually all of the problems associated with IL-2 administration. Many oncologists, comfortable with severe and prolonged neutropenia and thrombocytopenia, seem unwilling to contend with the physiologic disturbances caused by high-dose IL-2 administration. Decisions about the use of IL-2 seem to be more related to the training of oncologists than to the effectiveness of the treatment.

IL-2 should be the first-line treatment for patients with metastatic RCC. Most patients would not refuse to undertake this treatment if they were informed that it was the only chance of eradicating their disease and that the benefits far outweighed the risks. Sorafenib and sunitinib are valuable adjuncts for the treatment of these patients, but should most appropriately be used as second-line agents. 